\title{
Material properties and characterizations of cross-linked electro-spinning raspberry ketone incorporated polyvinyl alcohol/gelatin fibrous scaffolds
}

\author{
Trinh Quang Bao, Rose Ann Franco, Byong Taek Lee
}

Department of Biomedical Engineering and Materials, College of Medicine, Soonchunhyang University, Cheonan, Chungnam, Korea.

Email: $\underline{1 b t @ s c h . a c . k r}$

Received 20 October 2010; revised 26 October 2010; accepted 29 October 2010.

\begin{abstract}
The properties of polyvinyl alcohol/gelatin (PVA/GE) nanofibers have been previously investigated as a function of the processing parameters such as the ratios of PVA and GE, electrical field and tip-to-collector distance during the electro-spinning process, in this study, the properties of the electro-spinning PVA/GE nanofibers were examined when different solution feed rates were used to create the fibrous scaffold. The optimal conditions for the PVA/GE fibrous scaffold were determined to be a PVA/GE blend ratio of $8 / 2$, electrical field of $24 \mathrm{kV}$, tip-to-collector distance of $10 \mathrm{~cm}$ and speed rate of $1 \mathrm{ml} . \mathrm{h}^{-1}$. Using these conditions, Raspberry ketone (RK) was incorporated into PVA/GE fibrous scaffolds and their microstructure and material properties were characterized by SEM, DSC and XRD techniques. When the incorporated RK and PVA/GE fibrous scaffolds were crosslinked, the tensile strength and water-resistant ability increased at increasing cross-linking time. However, in the in vitro analysis, a longer cross-linking time was shown to increase its cytotoxicity. The cytotoxicity of RKPVA/GE-8 fibrous scaffold was evaluated based on a cell proliferation study by culturing L-929 fibroblast cell on the fibrous scaffold for 1,3 and 5 days. In these experiments, cell expansion was observed and the cells spread during the entire cell culture time.
\end{abstract}

Keywords: Electro-Spinning; PVA/GE Fibrous Scaffold; Raspberry Ketone; Nanofibers

\section{INTRODUCTION}

The electro-spinning (ES) method is the simplest method for manufacturing nanoscale fibrous scaffolds [1]. This process can be used to produce continuous fibers since this approach allows the scaffold to decrease the diameter of the pore size from micrometer to nanometer range [2]. Therefore, ES can be used to fabricate natural polymers, synthetic polymers, and polymer loaded with nanoparticles as well as metals and ceramics to enhance cell attachment and proliferation [3-6]. Furthermore, this technique is suitable for biomedical applications, such as the development of tissue engineering scaffolds, drug delivery systems, wound healing and wound dressing [7-10].

It is well known that GE derived from denatured collagen is suitable for cell adhesion. GE can form direct molecular interactions with cells to promote attachment and growth $[11,12]$. Hence, it has been widely used in the biomedical field as a sealant for vascular prostheses, dressings for wound healing and carriers for drug release [13]. However, GE is much less effective in fiber processing; thus, fabrication of practical microfibers containing GE through conventional ES is not common [14].

PVA is a non-toxic, biocompatible, and biodegradable synthetic polymer that has been broadly used in the biomedical field. Moreover, it has been extensively employed as a drug and protein carrier for the treatment of injuries and the regeneration of tissue because of its excellent properties and simple incorporation of drugs or proteins inside the fibers [15-17]. Furthermore, it is frequently combined with natural molecules that are recognized by cells favoring preliminary adhesion $[18,19]$.

$\mathrm{RK}$ is a compound found in red raspberries and has been used in medicine. Recently, some studies have reported that RK has anti-inflammatory activity when applied to the skin $[20,21]$. Due to these promising characteristics, RK was incorporated in a PVA/GE fibrous scaffold, which offers a unique combination of the inherent properties of electro-spun scaffolds and the anti-inflammatory activity for pain relief and healing.

In recent years, some studies have examined blends of 
PVA and GE in the form of PVA/GE films or PVA/GE sponges for industrial and tissue engineering applications $[18,22]$. In addition, Yang et al reported that the PVA/GE nanofibers have potential application in the controlled release of RK, which depended on the crosslinking time [21]. More recently, we studied the properties of PVA/GE nanofibers. In this previous study, the effect of different ratios of PVA/GE on the tensile strength of nano membranes was examined in detail [23]. However, no study has examined the effect of cross-linking time on the tensile strength and biocompatibility of PVA/ GE fibrous scaffold for the applications in a wound dressing device. There have been some reports that attempted to improve the tensile strength of GE nanofibers [11], PVA/GE nanofibers [21] by the cross-linking with glutaraldehyde (GTA). Thus, here, GTA was used to cross-link PVA/GE fibrous scaffold for different crosslinking times and biocompatibility of the cross-linked PVA/GE fibrous scaffold were investigated.

In this study, the effects of different solution feed rates on the morphology of the PVA/GE fibrous scaffolds were investigated by SEM. Based on these morphologic evaluations, the best conditions to fabricate RK incorporated PVA/GE fibrous scaffolds were determined. In addition, a GTA solution was used to cross-link the fibrous scaffolds and the properties of the cross-linked scaffolds were characterized. After the fibrous scaffolds were cross-linked, the mechanical properties, water-resistant ability and biocompatibility were examined as a function of the cross-linking time.

\section{MATERIALS AND METHODS}

\subsection{Materials}

PVA was obtained from Aldrich Chemical Co (USA). GE Type A (Approx. 300 Bloom, Sigma, St. Louis, MO) was obtained in powder form. RK was purchased from Sigma Co.; GTA was obtained from DeaJung Co.; Fetal bovine serum (FBS), P.S. (penicillin/streptomycin (antibiotics)), Dulbecco's phosphate buffered saline (D-PBS) without calcium or magnesiumand and MTT solution and trypsin-EDTA were purchased from GIBCO (Carlsbad, CA). The L-929 cell line was obtained from the ATCC Cell Line (CCL-1TM, NCTC clone 929 [L cell, L-929, derivative of Strain L], Korea). All other chemicals and solvents were in the analytical reagent grade.

\subsection{Preparation of Polymer Solutions, ES Setting and Cross-Linking Process}

Aqueous PVA solutions $(10 \% \mathrm{~m} / \mathrm{v})$ were prepared by dissolving PVA in deionized water at $80^{\circ} \mathrm{C}$ with constant stirring for 2 hours. The GE solution $(10 \% \mathrm{~m} / \mathrm{v})$ was also prepared by dissolving GE in an acetic acid solution at room temperature.
The GE solution was added into the PVA solution with specific volumes to obtain the PVA/GE (8/2) solution. Then, RK was added and the blend was mixed to obtain a homogenous solution. This solution was placed into a $10 \mathrm{ml}$ syringe fitted to a needle with a tip diameter of $21 \mathrm{GA}$, a syringe pump (lure-lock type, Korea) for controlling feed rates, and a grounded cylindrical stainless steel that was used to collect the fibers. The ES voltage was applied directly using a high DC voltage power supply (NNC-30 kilovolts-2 mA portable type, Korea).

The PVA/GE fibrous scaffolds were hung on the edge of the beaker. GTA (2\%) was added into the beaker and covered with aluminum foil for cross-linking through the evaporation of GTA. At predetermined intervals, the sample was taken out and dried for future studies.

\subsection{Characteristic Morphology and Analysis of Material Properties}

The morphology of the fibrous scaffolds was examined by scanning electron microscopy (SEM, JSM-7401F Japan). Differential Scanning Calorimetry (DSC) measurements (METTLER TOLEDO KOREA-DSC822e) were acquired with a sample weight of $3 \mathrm{mg}$ under nitrogen atmosphere. The fibrous scaffolds were subjected to X-ray Diffraction (XRD) (Rigaku, D/MAX-2500 V Japan) with $\mathrm{CuKa}$ radiation of $40 \mathrm{kV}$ and $200 \mathrm{~mA}$. The mechanical properties of the electrospun scaffolds were determined by using a universal testing machine $(\mathrm{R} \& \mathrm{~B}$ UNITECH-T) [4]. For water resistant ability test, the cross-linked PVA/GE scaffolds were cut into dimensions of $2 \times 2 \mathrm{~cm}^{2}$ then immersed into deionized water $\left(37^{\circ} \mathrm{C}\right)$ in incubator for a certain period of time before being dried to observe the morphology by SEM.

\subsection{In Vitro Study}

The cellular viability of fibroblast cells on samples was determined by using the MTT assay. The L-929 mouse fibroblast cells were seeded in 96-well tissue culture plates at 1,000 cells/well in $100 \mu \mathrm{l}$ RPMI. All media contained $10 \%$ of FBS and cell lines were incubated overnight. Diluted extract solutions of every fibrous scaffold at various concentrations $(0,12.5,25,50$ and $100 \%)$ were then added. The cells were treated for 1,2 , and 3 days and then $20 \mu \mathrm{l}$ of filtered MTT solution was added. After incubation at $37^{\circ} \mathrm{C}$ for $3 \mathrm{~h}$, the medium was removed from the well and $150 \mu \mathrm{l}$ of DMSO was added to dissolve any insoluble formazan crystals. The absorbance was measured at $560 \mathrm{~nm}$ using an ELISA reader. The cell viability was calculated as a percentage relative to the untreated control cells.

To examine the proliferation and spreading behavior of the cells, samples $20 \mathrm{~mm} \times 20 \mathrm{~mm}$ in size were steril- 
ized with $70 \%$ of EtOH for 30 minutes, then washed with PBS and suspended in conditioning medium (15 min). The fibroblast cells (L-929) were cultured on TCPs (Tissue Culture Polystyrene), control-like and samples. The cells were seeded at a cell density of $10^{4} \mathrm{cells} / \mathrm{cm}^{2}$ in RPMI and cultured for 1,3 and 5 days $\mathrm{s}$ at $37^{\circ} \mathrm{C}$ in a humidified air atmosphere with $5 \%$ of $\mathrm{CO}_{2}$.

\section{RESULTS}

\subsection{Morphology of Fibrous Scaffold from Electro-Spun}

Figure 1 shows SEM images of the PVA/GE nanofibers prepared from electro-spinning at various feed rates. The applied PVA/GE blend ratio, electric field and tip-tocollector were fixed at $8 / 2,24 \mathrm{kV}$ and $10 \mathrm{~cm}$, respectively. The diameter distributions and density distributions of the fibers at each different feed rate are shown in Figures 1(a), (b) and (c). Based on the analysis of the SEM images, the nanofibers were homogenous and uniform over a large area. However, there were differences in the fibers diameter and fibers density distribution be- tween the different samples. For example, at an applied feed rate of $0.05 \mathrm{ml}^{-1}{ }^{-1}$ (Figure 1(a)), the fibers were very thin and some of fibers gathered at the bead positions and were far from each other. In contrast, when the applied feed rate was $0.5 \mathrm{ml} . \mathrm{h}^{-1}$ (Figure 1(b)), the fibers were cylindrical, thicker, strained, non-woven and had a close distribution. On the other hand, when the applied feed rate was increased to $1 \mathrm{ml} \cdot \mathrm{h}^{-1}$, the fibers were very stretched, had a wide distribution and many small pore size were formed (Figure 1(c)). Beside the diameter of resulting fibers ranged from 40 to $170 \mu \mathrm{m}$.

Figure 2 shows the effect of incorporating RK on the morphology of the PVA/GE fibrous scaffold. The continuous fibers were successfully electro-spun from the PVA/GE solution. They possessed the common features of being round-shaped, randomly arrayed and highly porous. Both RK-free and RK incorporated PVA/GE fibers appeared smooth and no RK crystals were detected on the polymer surface. Figure 2(a) shows SEM photographs of the electro-spinning fiber without the incorporation of RK. Under these conditions the fibers were cylindrical, smooth and separated from each other.

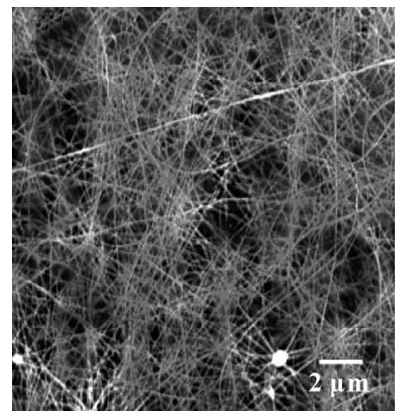

(a)

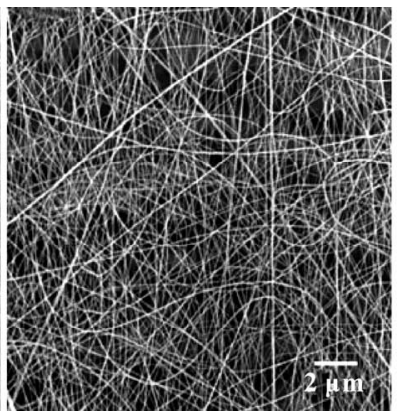

(b)

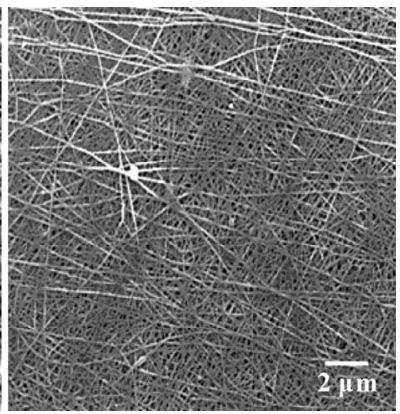

(c)

Figure 1. SEM images of PVA/GE fibers at a PVA/GE blend ratio of $8 / 2$, electric field of 24 $\mathrm{kV}$ and tip-to collector distance of $10 \mathrm{~cm}$ at feed rate of $0.05 \mathrm{ml}^{-1}$ (a), $0.5 \mathrm{ml}^{-1} \mathrm{~h}^{-1}$ (b) and 1 $\mathrm{ml} \cdot \mathrm{h}^{-1}(\mathrm{c})$.

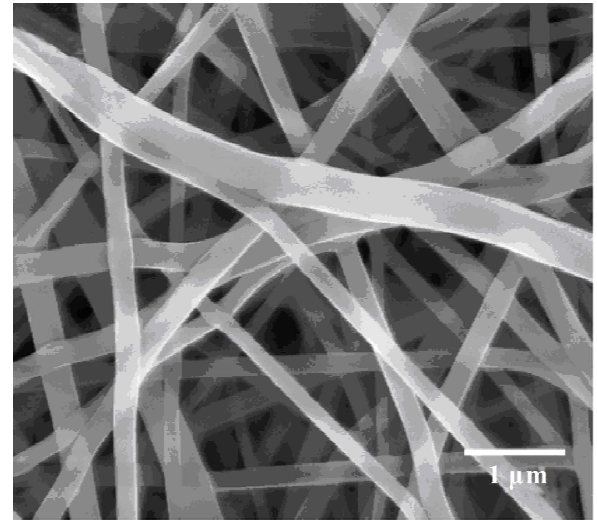

(a)

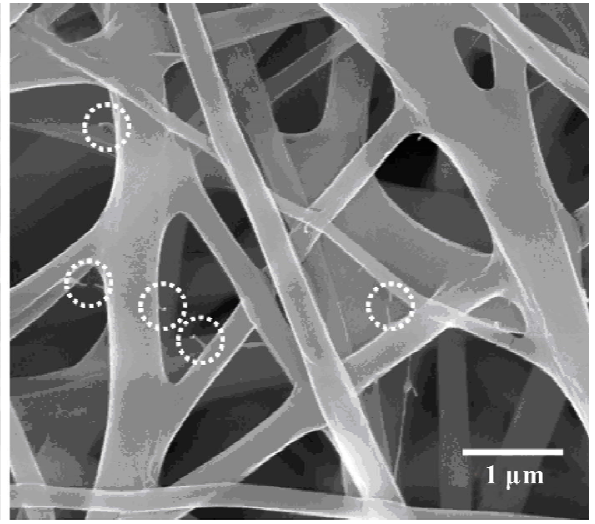

(b)

Figure 2. SEM images of PVA/GE fibrous scaffolds before (a) and after (b) RK incorporation. 
However, the morphology and average diameter of electro-spun nanofibers change significantly after RK was incorporated. Figure 2(b) shows that the fibers were attached and had melted into each other. In the case of RK incorporated PVA/GE, some of the stump-like structures were observed in the white circles. The average diameter of the RK-incorporated and RK-free fibers was $200 \pm 105,160 \pm 91 \mathrm{~nm}$, respectively. These results demonstrate that incorporation of RK significantly altered the diameter of the nanofiber.

\subsection{X-Ray Diffraction}

Figure 3 shows the XRD patterns of RK, PVA/GE fibrous scaffold and RK incorporated PVA/GE fibrous scaffold. Several distinct peaks were observed for RK at diffraction angles of $2 \theta=14.4^{\circ}, 15.6^{\circ}, 20.4^{\circ}, 23.7^{\circ}, 23.9^{\circ}$, $28.6^{\circ}$ and $34.8^{\circ}$. The diffraction of RK incorporated PVA/GE fibrous scaffold exhibited several peaks at $2 \theta=$ $14.5^{\circ}, 15.7^{\circ}, 19.4^{\circ}, 20.2^{\circ}, 20.4^{\circ}, 23.7^{\circ}, 24.1^{\circ}, 26.4^{\circ}, 28.3^{\circ}$, and $27.9^{\circ}$. While the PVA/GE fibrous scaffold had a broad peak at $2 \theta=20.9^{\circ}$. In addition, some peaks such as $2 \theta=34.8^{\circ}$ of RK and $2 \theta=20.9^{\circ}$ of PVA/GE fibrous scaffold were not observed in the XRD pattern of the RK incorporated PVA/GE fibrous scaffold. These results indicate that the fibrous scaffold structure of RK incorporated PVA/GE changes relative the structure of RK and the PVA/GE fibrous scaffold alone.

\subsection{Differential Scanning Calorimetry (DSC)}

DSC thermograms of the RK and RK incorporated PVA/ GE fibrous scaffold are shown in Figure 4. The pure RK showed a relatively sharp endothermic curve with a peak at $67^{\circ} \mathrm{C}$. However, two peaks were observed in the DSC scan of RK incorporated PVA/GE fibrous scaffold, which corresponded to the melting of the crystal structure of $\mathrm{RK}$ at $105^{\circ} \mathrm{C}$ and $\mathrm{PVA} / \mathrm{GE}$ matrix at $225^{\circ} \mathrm{C}$. The observed melting peaks of RK incorporated PVA/GE fibrous scaffold were much different from that of pure RK $\left(67^{\circ} \mathrm{C}\right)$ and PVA/GE fibrous scaffold $\left(100^{\circ} \mathrm{C}\right)$ [23], which implies that particular interactions were present between RK and the PVA/GE matrix in RK incorporated PVA/GE fibrous scaffold.

\subsection{Mechanical Properties}

Figure 5 shows the stress-strain curves of RK incorporated PVA/GE fibrous scaffolds at different cross-linking times. Based on the stress-strain curves, respective tensile properties in terms of tensile strength and strain at break are summarized in Table 1.

The tensile results indicated that cross-linking improved the mechanical performance of the RK incorporated PVA/GE fibrous scaffolds. The tensile strength of RK incorporated PVA/GE fibrous scaffold increased as

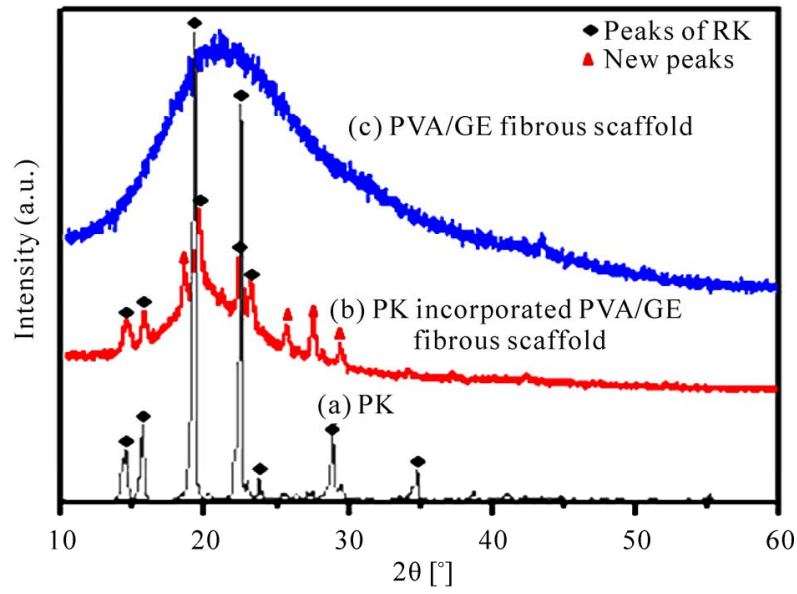

Figure 3. XRD profile of RK (a), RK incorporated PVA/GE fibrous sc fold (b) and PVA/GE fibrous scaffold (c).

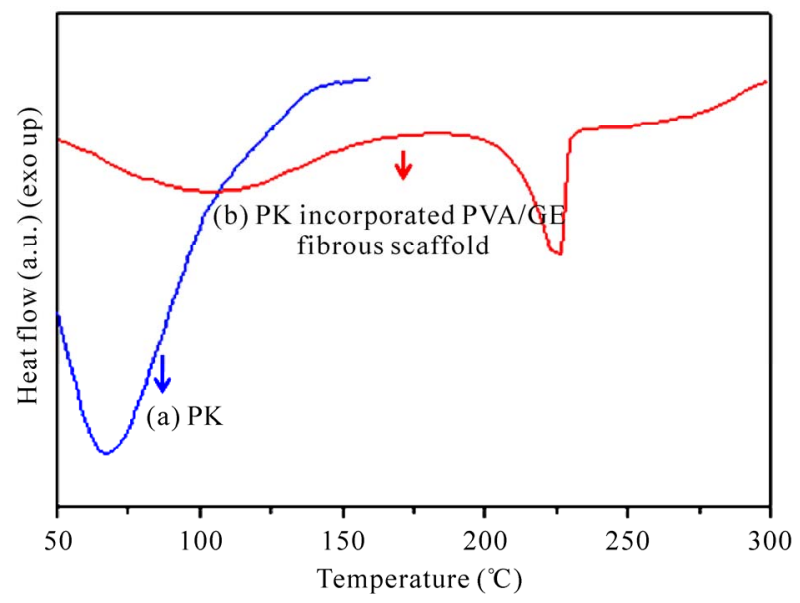

Figure 4. DSC spectra of RK (a) and RK incorporated PVA/ GE fibrous scaffold (b).

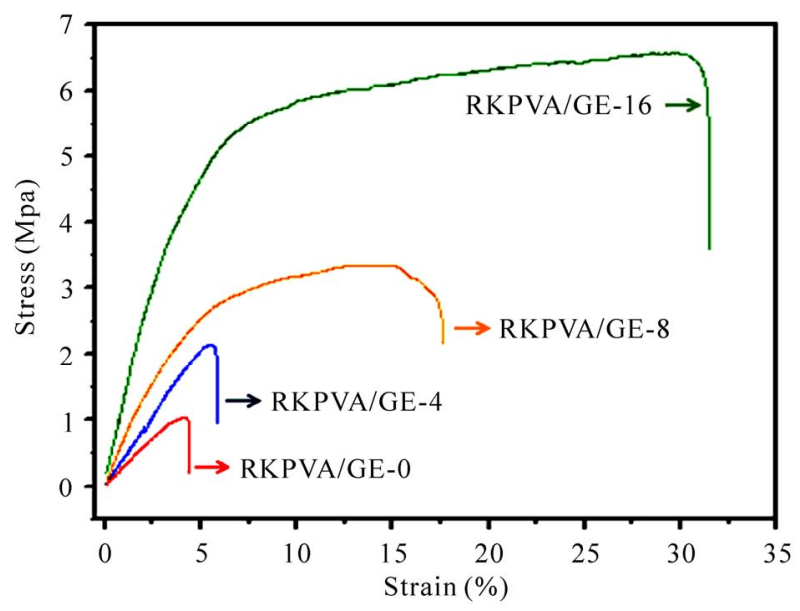

Figure 5. Stress-strain curves of RK incorporated PVA/GE fibrous scaffolds after 0 hour (RKPVA/GE-0), 4 hours (RKPVA/ GE-4), 8 hours (RKPVA/GE-8) and 16 hours (RKPVA/GE-16) of cross-linking. 
the cross-linking time increased.

\subsection{SEM Morphology of Fibrous Scaffolds after Water-Resistant Ability Testing}

After RK incorporated PVA/GE fibrous scaffolds were treated in GTA vapor for $0,4,8$ and 16 hours, their water-resistant behaviors were evaluated and summarized in Table 2. In these experiments, samples untreated, treated up to 4 and 8 hours were found to be totally dissolved in $37^{\circ} \mathrm{C}$ water after being immersed for 1,2 and 3 days, respectively. In contrast, the sample cross-linked for 16 hours remained intact after immersion in water at $37^{\circ} \mathrm{C}$.

SEM images of the scaffolds at different cross-linking times are shown in Figure 6. Based on the analysis of the SEM images, many break points were observed in the fiber when not treated with GTA (Figure 6(a)). After the cross-linking time was increased to 4 hours, the fibers were devoid of break points, and the fibers appeared smooth with less points of erosion (Figure 4(b)). In the case of 8 hours of cross-linking (Figure 1(c)), the surface of the fibers appeared homologous and uniform and some pores were clearly visible. However, when the
Table 1. Tensile properties of RK incorporated PVA/GE fibrous scaffold depending on cross-linking times.

\begin{tabular}{ccc}
\hline Samples & Tensile strength (M Pa) & Strain at break (\%) \\
\hline RKPVA/GE-0 & $1.0 \pm 0.4$ & $4.2 \pm 0.2$ \\
RKPVA/GE-4 & $2.1 \pm 0.9$ & $5.7 \pm 0.8$ \\
RKPVA/GE-8 & $3.3 \pm 0.7$ & $17.6 \pm 1.6$ \\
RKPVA/GE-16 & $6.2 \pm 0.6$ & $30.1 \pm 2.3$ \\
\hline
\end{tabular}

Table 2. Effect of cross-linking time on water resistant ability of PVA/GE fibrous scaffolds.

\begin{tabular}{ccccc}
\hline \multirow{2}{*}{$\begin{array}{c}\text { Immersion time } \\
\text { at 37 } \mathbf{C}\end{array}$} & \multicolumn{4}{c}{ Time of cross-linking } \\
\cline { 2 - 5 } & 0 hour & 4 hours & $\mathbf{8}$ hours & $\mathbf{1 6}$ hours \\
\hline Day 1 & $\mathrm{Y}$ & $\mathrm{Y}$ & $\mathrm{Y}$ & $\mathrm{Y}$ \\
Day 2 & $\mathrm{N}$ & $\mathrm{Y}$ & $\mathrm{Y}$ & $\mathrm{Y}$ \\
Day 3 & & $\mathrm{N}$ & $\mathrm{N}$ & $\mathrm{Y}$ \\
\hline
\end{tabular}

Y denotes samples remained and $\mathrm{N}$ denotes sample dissolved.

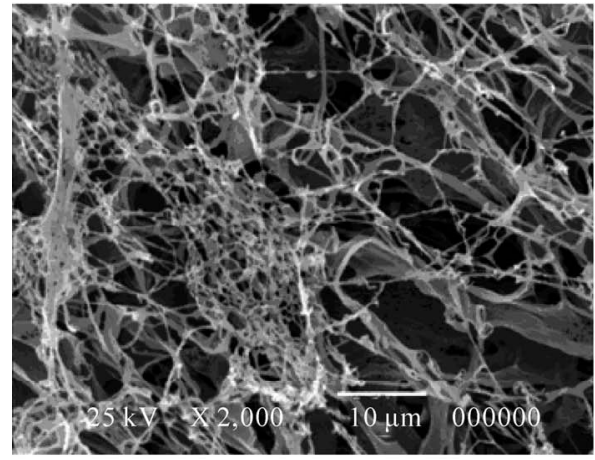

(a)

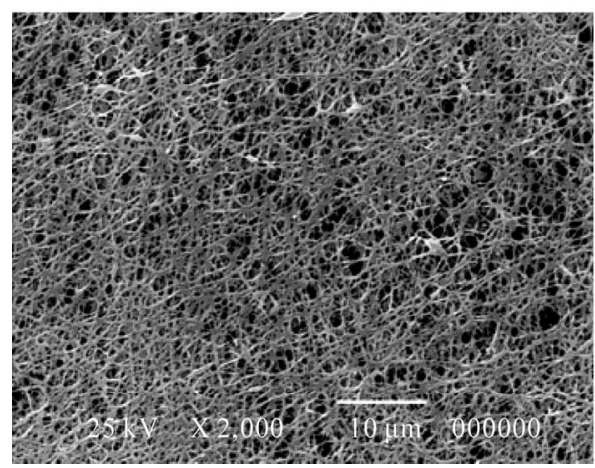

(c)

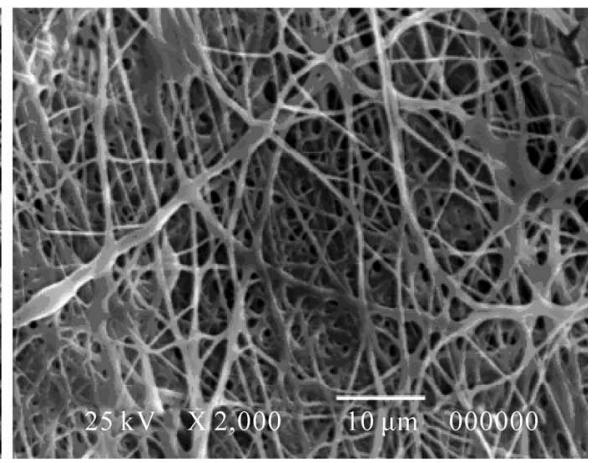

(b)

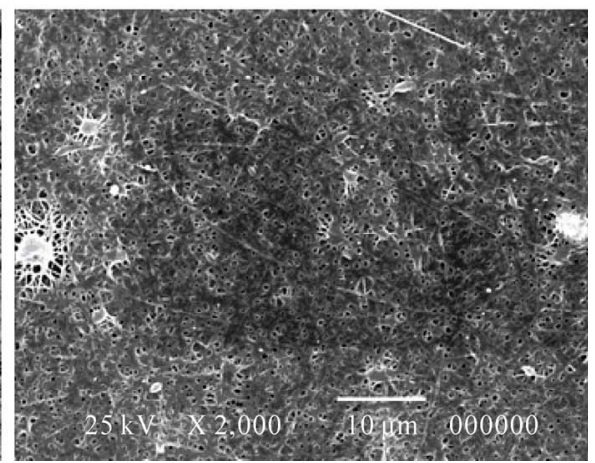

(d)

Figure 6. SEM images of RK incorporated PVA/GE fibrous scaffolds at different cross-linking times: 0 hour (a), 4 hours (b), 8 hours (c) and 16 hours (d) after immersion in water at $37^{\circ} \mathrm{C}$ for one day. 
samples were cross-linked for 16 hours (Figure 6(d)), the fibers appeared to adhere to each other. The surface of the scaffold was dense and contained many small pores. The results of these experiments demonstrated that the cross-linking treatment improved the water-resistant of electro-spun of RK incorporated PVA/GE fibrous scaffolds.

\subsection{Cytotoxicity Results}

Figure 7 shows the cytotoxicity results of RK incorporated PVA/GE fibrous scaffolds after $0,4,8$ and 16 hours cross-linking by quantitative analysis using the MTT test [24]. After allowing the cells to spread for 3 days at various extract dilutions $(0,12.5,25,50$ and $100 \%$ ), the proliferation of L-929 cells was measured using the MTT assay. In this analysis, we found that the cells proliferated better on RKPVA/GE-8 than on RKPVA/ GE-16 scaffolds. However, the proliferation of cells was significantly higher on RKPVA/GE-0 compared to both RKPVA/GE-4 and RKPVA/GE-8 scaffolds. Based on these results, it appears that the extracts of the scaffolds displayed no cytotoxic reactivity in this test and cell metabolism of RK incorporated PVA/GE fibrous scaffolds decreased with an increase in the cross-linking time.

\subsection{SEM Morphology of Cell Proliferation and Spreading}

The cell morphology of RKPVA/GE-8 fibrous scaffold was examined by SEM at days 1, 3 and 5, and the results are shown in Figure 8. It was estimated that at day 1 (Figures 8(a) and (b)) L-929 fibroblast cells only attached to the surfaces through discrete filodia. After just 3 days, the shape of the fibroblast cells changed from round to elongated (Figures 8(b) and (c)), they stretched across the nanofibrous substrates and increased in numbers. Subsequently, L-929 fibroblast cell proliferation and growth continued progressively, and by day 5 (Figure 8(d)) the cells had increased significantly in numbers. Thus, the RKPVA/GE-8 scaffold was almost completely covered with a continuous L-929 fibroblast cell monolayer. These SEM images demonstrated that the L-929 fibroblast cells successfully proliferated and spread on RKPVA/GE-8. Based on these results, the RKPVA/ GE-8 fibrous scaffold has promise for use in biomedical applications.

\section{DISCUSSION}

The effects of electro-spinning parameters, such as polymer concentration, electrical field and tip-to-collector distance on the morphology of nanofibers was previously investigated [3,23]. In addition, Duppi et al. [5] examined the effect of feed rate and reported that an

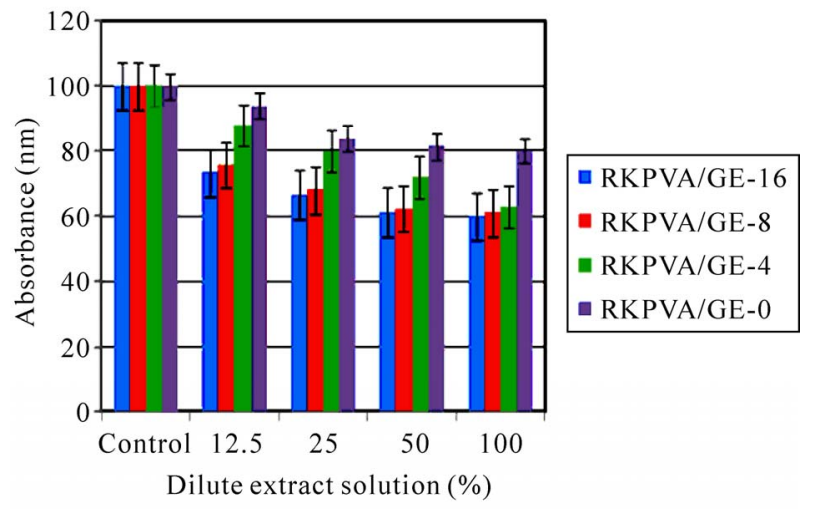

Figure 7. The cytotoxicity of RK incorporated PVA/GE fibrous scaffolds after 0 hour (RKPVA/GE-0), 4 hours (RKPVA GE-4), 8 hours (RKPVA/GE-8) and 16 hours (RKPVA/GE-16) of cross-linking.

increase solution feed rate could increase the fiber diameter, when the jet is properly stretched by the electric force, or cause the formation of beads. The results of our work are consistent with that study and the SEM images shown in Figure 1 clearly demonstrates that the diameter of the PVA/GE nanofiber significantly increased when the solution feed rate was increased from $0.05 \mathrm{ml}^{-1} \mathrm{~h}^{-1}$ to 1 $\mathrm{ml} . \mathrm{h}^{-1}$. Combined with our previous study [23], we produced a homogeneous, non-woven and fine PVA/GE fibrous scaffold by using a PVA/GE blend ratio of $8 / 2$, electrical field of $24 \mathrm{kV}$, tip-to-collector distance of 10 $\mathrm{cm}$, and solution feed rate of $1 \mathrm{ml} \cdot \mathrm{h}^{-1}$. An acetic acid -water solution was used as a solvent for the electro-spun PVA/GE fibrous scaffolds.

RK was incorporated into electro-spun PVA/GE nanofibers because of its biological effects in influencing cell behavior. Incorporation of RK did not significantly influence the morphology of the resulting fibers as the both RK-free and RK-loaded composite fibers remained unaltered, microscopically and uniform nanofibers (Figure 2) with an average fiber diameter higher than that of their analogous unloaded system were observed. The formation of a uniform fiber and fibrous structure, reflected the high conductivity of acetic acid [23] and the stability of the applied optimized conditions of electro-spinning. However, after RK incorporation, some stump-like structures were observed, which was due to the fragility of the polymer fibers [25]. On the other hand, RK possesses a crystal structure at room temperature. The structure of RK in the PVA/GE matrix and acetic acid applying under the high electrical potential that was used here in electro-spinning is still unknown. To determine if the structure of RK was altered under these conditions, RK incorporated PVA/GE fibrous scaffold were investigated by XRD and DSC. Figure 3 shows the XRD profile of RK, PVA/GE fibrous scaffold and RK 


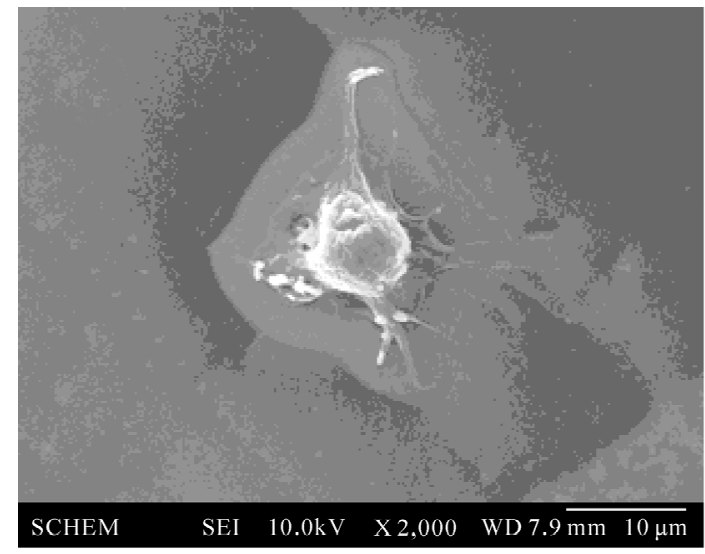

(a)

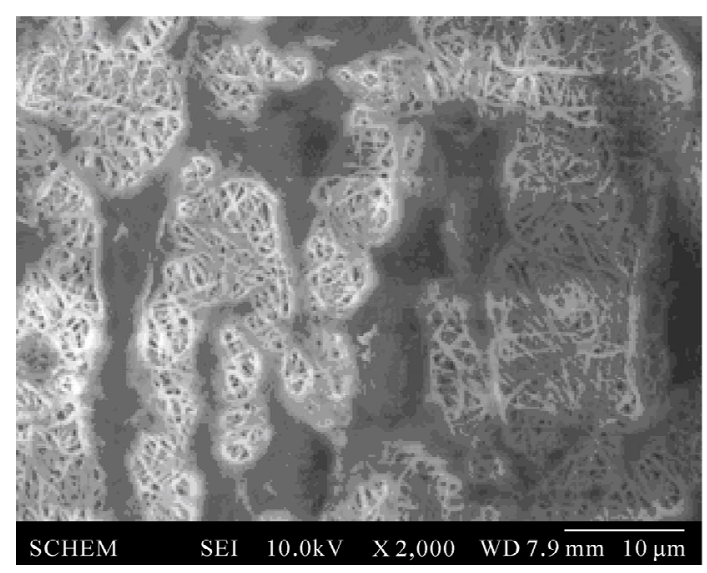

(c)

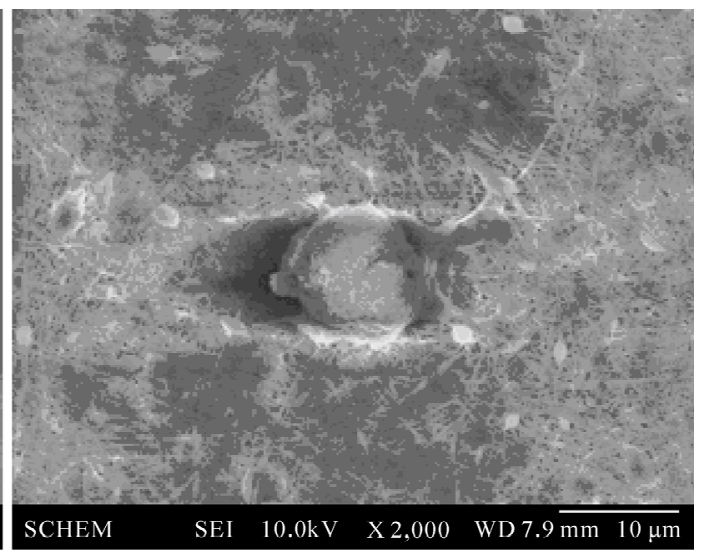

(b)

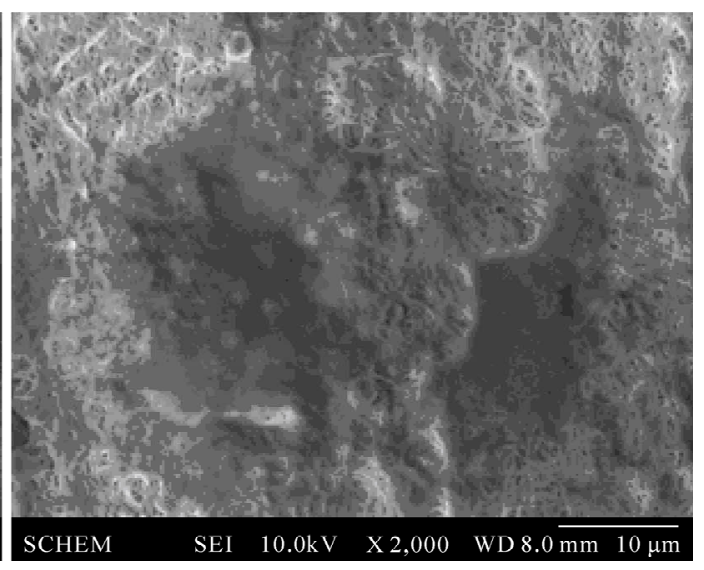

(d)

Figure 8. SEM images of L-929 fibroblast cell on RKPVA/GE-8 fibrous scaffold after 1, 3 and 5 days of culture cell: control sample (a), 1 day (b), 3 days (c) and 5 days of culture cell (d).

incorporated PVA/GE fibrous scaffold. In this analysis, the structural integrity of RK in RK incorporated PVA/ GE fibrous scaffold was not preserved, since peaks corresponding to RK disappeared and some new peaks were observed in the XRD profile of RK incorporated PVA/ GE fibrous scaffold. Based on these results, we believe that strong interactions occur between PVA/GE and RK in the acetic acid environment. The results from XRD were supported by DSC analysis. In addition, the sharp melting peak of RK was shifted toward a high temperature and the melting peak became broader (from $67^{\circ} \mathrm{C}$ to $105^{\circ} \mathrm{C}$ ), as shown in Figure 4 . These results confirmed the crystal structure of RK changed when it was incorporated into the PVA/GE fibrous scaffold.

Yang et al. [21] previously reported that RK, PVA and GE are water-soluble molecules. Therefore, when coming into contact with an aqueous medium, RK incorporated PVA/GE fibrous scaffold may partially dissolve and it may lose its fibrous structure upon exposure to high ambient humidity for a certain period of time. To increase the stability of RK incorporated PVA/GE fibrous scaffold in applications that require exposure to an aqueous medium or high humidity, further cross-linking is necessary. Figure 5 shows that the tensile strength and strain at break of RK incorporated PVA/GE fibrous scaffolds increased as the cross-linking time was increased. The results from the water-resistant ability tests demonstrated that the degree of cross-linking significantly impacted its water resistance. It was found that the grossly degree was increased as the cross-linking time was increased and similarly the density of the surface of the fibrous scaffold was also increased, which was demonstrated by the fact that the fibers at junctions were fused together through bonds. When the RK incorporated PVA/GE fibrous scaffold were cross-linked for 16 hours they were found to still be water resistant after 3 days of incubation. Therefore, a longer crosslinking time increased the mechanical properties and improved the water resistant ability of RK incorporated PVA/GE fibrous scaffolds. However, it has been re- 
ported that the chemically cross-linked biomaterials, un-reacted cross-linking agents and leached material during degradation may be cytotoxicity [26]. Figure 7 shows that the cytotoxicity of the RK incorporated PVA/GE fibrous scaffolds increased as the cross-linking time increased. Based on these combined results, RK incorporated PVA/GE fibrous scaffold after cross-linked for 8 hours (RKPVA/GE-8) was chosen for subsequent in vitro experiments to evaluate the proliferation and spreading of L-929 fibroblast cells after 1, 3 and 5 days culture cell. Figure 8 shows the proliferation and spreading of fibroblast cells, as well as its interaction with the RKPVA/GE-8 over a cell culture period of 5 days. Carroll [27] reported that the insulin-like growth factor-I (IGF-I) has various important biological effects such as promoting differentiation of various cell types, potent anti-apoptotic activity, and an anabolic effect. Furthermore, Harada et al. [28] reported that RK might increase dermal IGF-I production through neuron activation, thereby promoting hair growth and increasing skin elasticity. The PVA/GE fibrous scaffolds have a large surface area, non-woven fibers, randomly arrayed fibers and is highly porous, all of which may have promoted cell proliferation and spreading on RKPVA/GE- 8 after 5 days of culture. Thus, this scaffold could be used to promote faster restoration and increase the biocompatibility of wound dressings.

\section{CONCLUSION}

PVA/GE fibrous scaffolds were successfully fabricated by the ES method. In this process, different solution feed rates were shown to affect the morphology of nanofibers. XRD and DSC analysis demonstrated that the RK crystal structure changed when incorporated in the PVA/GE fibrous scaffold. The mechanical property, water resistant ability, and cytotoxicity of the fibrous scaffolds increased as the cross-linking time increase. The in vitro analysis demonstrated that RK incorporated PVA/GE fibrous scaffold after 8 hours of cross-linking (RKPVA/ GE-8) were highly biocompatible. Therefore, RKPVA/ GE-8 holds promise for use in tissue engineering and wound dressing devices.

\section{ACKNOWLEDGEMENTS}

This work was supported by Mid-career Research Program through NRI grant funded by the MEST (2009-0092808).

\section{REFERENCES}

[1] Andreas, G. and Joachim, H.W. (2007) Electrospinning: A fascinating method for the preparation of ultrathin fibers. Angewandte Chemie International Edition, 46, 5670-5703. doi:10.1002/anie.200604646

[2] Supaphol, P. and Chuangchote, S. (2008) On the electro- spinning of poly (vinyl alcohol) nanofiber mats: A revisit. Journal of Applied Polymer Science, 108, 969-978. doi:10.1002/app.27664

[3] Bhattarai, N., Edmondson, D., Veiseh, O., Matsen, F.A. and Zhang, M. (2005) Electrospun chitosan-based nanofibers and their cellular compatibility. Biomaterials, 26, 6176-6184.

[4] Nguyen, T.H., Lee, K.H. and Lee, B.T. (2010) Fabrication of Ag nano particles dispersed in pva nanowires mats by microwave irradiation and electro-spinning. Materials Science and Engineering C, 30, 944-950. doi:10.1016/j.msec.2010.04.012

[5] Puppi, D., Piras, A.M., Detta, N., Dinucci, D. and Chiellini, F. (2010) Poly (lactic-co-glycolic acid) electrospun fibrous meshes for the controlled release of retinoic acid. Acta Biomaterialia, 6, 1258-1268.

[6] John, M.J. and Thomas, S. (2008) Biofibres and biocomposites. Carbohydrate Polymers, 71, 343-364.

[7] Li, W.J., Laurencin, C.T., Caterson, E.J., Tuan, R.S. and Ko, F.K. (2002) Electrospun nanofibrous structure: A novel scaffold for tissue engineering. Journal of Biomedical Materials Research Part B: Applied Biomaterials, 60, 613-621.

[8] Chong, E.J., Phan, T.T., Lim, I.J., Zhang, Y.Z., Bay, B.H., Ramakrishna, S. and Lim, C.T. (2007) Evaluation of electrospun $\mathrm{PCL} /$ gelatin nanofibrous scaffold for wound healing and layered dermal reconstitution. Acta Biomaterialia, 3, 321-330. doi:10.1016/j.actbio.2007.01.002

[9] Rujitanaroj, P., Pimpha, N. and Supaphol, P. (2008) Wounddressing materials with antibacterial activity from electrospun gelatin fiber mats containing silver nanoparticles. Polymer, 49, 4723-4732.

[10] Chena, J.P., Chang, G.Y. and Chen, J.K. (2008) Electrospun collagen/chitosan nanofibrous membrane as wound dressing. Colloids and Surfaces A: Physicochemical and Engineering Aspects, 313, 183-188. doi:10.1016/j.polymer.2008.08.021

[11] Zhang, Y.Z., Venugopal, J., Huang, Z.M., Lim, C.T. and Ramakrishna, S. (2006) Crosslinking of the electrospun gelatin nanofibers. Polymer, 47, 2911-2917.

[12] Awad, H.A., Wickham, M.Q., Leddy, H.A., Gimble, J.M. and Guilak, F. (2004) Chondrogenic differentiation of adipose derived adult stem cells in agarose, alginate, and gelatin scaffolds. Biomaterials, 25, 3211-3222. doi:10.1016/j.biomaterials.2003.10.045

[13] Gaihre, B., Khil, M.S., Lee, D.R. and Kim, H.Y. (2009) Gelatin-coated magnetic iron oxide nanoparticles as carrier system: Drug loading and in vitro drug release study. International Journal of Pharmaceutics, 365, 180-189. doi:10.1016/j.ijpharm.2008.08.020

[14] Huang, Z.M., Zhang, Y.Z., Ramakrishna, S. and Lim, C.T. (2004) Electrospinning and mechanical characterization of gelatin nanofibers. Polymer, 45, 5361-5368. doi:10.1016/j.polymer.2004.04.005

[15] Zeng, H., Du, Y., Yu, J., Huang, R. and Zhang, L., (2001) Preparation and characterization of chitosan/poly (vinyl alcohol) blend fibers. Journal of Applied Polymer Science, 80, 2558-2565.

[16] Yang, D.Z., Long, Y.H. and Nie, J. (2008) Release of lysozyme from electrospun PVA/lysozyme-gelatin scaffolds. Frontiers of Materials Science in China, 2, 261265. doi:10.1007/s11706-008-0053-1 
[17] Ngawhirunpat, T., Opanasopit, P., Rojanarata, T., Akkaramongkolporn, P., Ruktanonchai, U. and Supaphol, P. (2009) Development of Meloxicam-loaded electrospun polyvinyl alcohol mats as a transdermal therapeutic agent. Pharmaceutical Development and Technology, 14, 70-79.

[18] Moscatoa, S., .Mattiia, L, D’Alessandroa, D., Casconeb, M.G., Lazzerib, L., Serinoa, L.P., Dolfia, A. and Bernardini, N. (2008) Interaction of human gingival fibroblasts with PVA/gelatine sponges. Micron, 39, 569-579. doi:10.1016/j.micron.2007.06.016

[19] Pham, Q.P., Sharma, U. and Mikos, A.G. (2006) Electrospinning of polymeric nanofibers for tissue engineering applications. Tissue Engineering, 12, 1197-1211. doi:10.1089/ten.2006.12.1197

[20] Chie, M., Yurie, S., Mariko, H., Shintaro, I., Takahiro, T. and Hiromichi, O. (2005) Anti-obese action of raspberry ketone. Life Science, 11, 194-204.

[21] Yang, D., Li, Y. and Nie, J. (2007) Preparation of gelatin/PVA nanofibers and their potential application in controlled release of drugs. Carbohydrate Polymers, 69, 538-543. doi:10.1016/i.carbpol.2007.01.008

[22] Chiellini, E., Cinelli, P., Fernandes, G.E., Kenawy, E.S. and Lazzeri, A. (2001) Gelatin-based blends and composites. Morphological and thermal mechanical characterization. Biomacromolecules, 2, 806- 811.

[23] Linh, N.T.B., Min, Y.K., Song, H.Y. and Lee, B.T. (2010)
Fabrication of polyvinyl alcohol/gelatin nanofiber composites and evaluation of their material properties. Journal of Biomedical Materials Research Part B: Applied Biomaterials, 95B, 184-191. doi:10.1002/jbm.b.31701

[24] Hiep, N.T. and Lee, B.T. (2010) Electro-spinning of PLGA/PCL blends for tissue engineering and their biocompatibility. Journal of Materials Science: Materials in Medicine, 21, 1969-1978.

[25] Han, J., Chen, T.X., White, C.J.B. and Zhu, L.M. (2009) Electrospun shikonin-loaded PCL/PTMC composite fiber mats with potential biomedical applications. International Journal of Pharmaceutics, 382, 215-221. doi:10.1016/j.ijpharm.2009.07.027

[26] Jayakrishnan, A. and Jameela, S.R. (1996) Glutaraldehyde as a fixative in bioprostheses and drug delivery matrices. Biomaterials, 17, 471-484.

[27] Carroll, P.V. (2001) Treatment with growth hormone and insulin-like growth factor-I in critical illness. Best Practice \& Research Clinical Endocrinology \& Metabolism, 15, 435-451. doi:10.1053/beem.2001.0162

[28] Harada, N., Okajima, K., Narimatsu, N., Kurihara and H., Nakagata, N. (2008) Effect of topical application of raspberry ketone on dermal production of insulin-like growth factor-I in mice and on hair growth and skin elasticity in humans. Growth Hormone \& IGF Research, 18, 335-344. doi:10.1016/j.ghir.2008.01.005 